Journal of Humanities and Social Sciences Studies (JHSSS)

ISSN: $2663-7197$

DOI: 10.32996/jhsss

Journal Homepage: www.al-kindipublisher.com/index.php/jhsss

\title{
The Impact of E-learning on Virtual Class Interactions: A Case Study of French Virtual Class Interactions
}

\author{
Omer Ahmed Omer ${ }^{1} \square$ and Nadra Abd Allah Ali ${ }^{2}$ \\ ${ }^{1}$ College of Sciences and Arts (Turaif), NBU, Saudi Arabia \\ ${ }^{2}$ Faculté des Lettres, Université de Khartoum, Soudan
}

$\square$ Corresponding Author: Omer Ahmed Omer, E-mail: Omar.Ahmed@nbu.edu.sa

ARTICLE INFORMATION ABSTRACT

Received: 10 October 2021

Accepted: 15 November 2021

Published: 30 November 2021

DOI: $10.32996 /$ jhsss.2021.3.11.12

\section{KEYWORDS}

E-learning, interaction, virtual class, structured observation
The current qualitative study aims at exploring and analyzing in a descriptive way the impact of e-learning on different interactions forms in French virtual class in order to strengthen the students' engagement in this type of class. The problem that we encounter when it comes to French virtual class is the lack of spontaneous and active interactions which could negatively affect the learning process. The study was based on data collected from structured observations conducted throughout the $2^{\text {nd }}$ semester 2020/2021. The results show that the general interaction rate is unsatisfactory; especially the peers' interaction rate which is the worst. To enhance the virtual class interactions, some tips and practices have been recommended. A further study may help to identify the factors that affect the interactions in virtual class.

\section{Introduction}

Aujourd'hui, nous vivons dans une situation exceptionnelle en raison de la crise sanitaire liée à la COVID-19, apparue vers la fin de l'année 2019. Comme nous le savons, la pandémie de grande ampleur dont les répercussions ressenties dans tous les secteurs de la vie (social, économique, éducatif, culturel, politique, etc.). Devant cette situation que le monde n'a jamais connue, les institutions scolaires et universitaires se sont fermées afin de réduire la propagation de l'infection virale et de protéger les étudiants, les enseignants et les employés d'être infectés par le virus. Suivant la fermeture des établissements scolaires et universitaires, beaucoup d'institutions ont eu le recours à la formation en ligne (terme recommandé en France ou l'apprentissage en ligne, terme recommandé au Canada ou encore l'e-learning) en tant qu'alternative à la formation traditionnelle dite présentielle. Dès lors, les cours se sont donnés en ligne. Ainsi, la formation en ligne est devenue une réalité vivante mise en place en vue d'assurer la continuité du processus d'apprentissage et de la formation. En effet, ce type de formation, qui fait partie de la formation ouverte et à distance (FOAD) comme nous le verrons plus tard, ne cesse de faire couler beaucoup d'encre depuis son émergence. Certains le considèrent comme une nécessité sociale qu'on doit l'intégrer dans le processus d'apprentissage tandis que d'autres le voient comme un luxe social. À l'ère de la Covid-19, la formation en ligne s'est imposée comme une réalité incontournable ce qui a engendré de nombreuses questions et préoccupations quant à son efficacité et sa rentabilité.

\section{Présentation du problème}

Le problème qui nous inquiète ici est relative à l'interaction active considérée comme un élément d'autant plus problématique chez certains apprenants au Département d'anglais, Faculté des Sciences et des Lettres, Campus de Turaif, Northern Border University, KSA. Ces apprenants s'enferment dans leur passivité et ne participent guère ce qui défavorise l'acquisition des capacités d'interagir et de communiquer. Les défis auxquels nous sommes confrontés se résument donc au niveau du manque quasi total d'interactions spontanée et active. Ce manquement est courant chez bon nombre d'étudiants ce qui aurait des effets négatifs sur l'acquisition des capacités des savoirs.

\section{Objectifs}

Dès qu'il est question de la crise sanitaire actuelle, la limitation des déplacements, voire le confinement vient à l'esprit comme mesures prises par plusieurs pays afin d'arrêter la propagation de la pandémie et d'assurer la continuité de la vie, y compris le

Copyright: (c) 2021 the Author(s). This article is an open access article distributed under the terms and conditions of the Creative Commons Attribution (CC-BY) 4.0 license (https://creativecommons.org/licenses/by/4.0/). Published by Al-Kindi Centre for Research and Development, London, United Kingdom. 
télétravail. En effet, le secteur de l'éducation n'étant pas à l'abri de cette crise. La majorité d'institutions scolaires et universitaires se sont tournés vers la formation en ligne, elles ont eu le recours à l'enseignement en distanciel rendu facile par les nouvelles technologies de l'information et de la communication pour assurer la continuité du processus d'apprentissage. Une formation qui se fait $100 \%$ en ligne est une nouvelle expérience étant en cours de développement et d'amélioration qui mérite d'être étudiée. Beaucoup de pays se sont adaptés à ce type de formation en tant que mode unique de formation ou un mode complémentaire au mode traditionnel dans le cadre de la lutte contre la Covid-19. Face à cette pratique relativement nouvelle, certaines voix sont élevées réclamant la nécessité d'étudier minutieusement l'expérience. De là est né notre intérêt qui consistait à faire une étude sur l'expérience vécue par nos étudiants et ses effets sur leur apprentissage, c'est-à-dire, nous voulons mettre en lumière les effets possibles de l'apprentissage en ligne sur les capacités d'interagir et de communiquer. En d'autres termes, notre intérêt se porte plus particulièrement sur l'interaction active et spontanée en classe virtuelle afin de renforcer et optimiser le rôle que jouent les capacités d'interaction active dans l'acquisition des savoirs interactionnels et communicationnels. La présente étude vise donc à évaluer l'impact de la formation en ligne sur les interactions spontanée et active pour relever les défis auxquels se heurte ce type de formation. Nous essaierons à la fin de cette étude d'apprécier le taux d'interactions dans nos classes virtuelles de FLE ce qui nous permettra, comme nous le souhaitons, de proposer certaines pratiques qui participent à renforcer l'interaction en classe virtuelle.

\section{Importance de l'étude:}

L'importance fondamentale de cette étude réside dans le fait que l'interaction en classe joue un rôle primordial dans le processus d'apprentissage que ce soit un apprentissage en présentiel ou en distanciel. II va sans dire que l'interaction est censée assurer, entre autres, la communication et l'échange d'idées entre les étudiants et l'enseignant ce qui favorise l'acquisition des habilités langagières et crée un climat social et émotionnel favorables. Partant de ce constat, nous estimons qu'une étude ayant pour objet l'appréciation de différents types d'interactions aurait une importance accrue notamment lorsqu'il s'agit de la formation en ligne, mode de formation globalement imposé suite à la propagation de la flambée pandémique de la COVID-19 ce qui permet, comme nous le souhaiterions, d'étudier minutieusement le comportement interactionnel de nos apprenants quant à une expérience formatrice relativement nouvelle.

\section{Cadre conceptuel}

\subsection{Définition de la formation à distance:}

La formation à distance, qui s'applique tant à la formation continue qu'à la formation initiale, connue sous plusieurs appellations dont la formation ouverte et à distance (FOAD), l'enseignement à distance (EAD), la formation en ligne, l'e-learning (Glikman 2002), n'est pas une nouvelle pratique dans la pédagogie. Ces diverses dénominations se rencontrent au concept général et aux finalités, mais elles se diffèrent aux modes de formation utilisés ce qui laisse à croire qu'il n'y a pas de consensus sur le terme quant à sa définition, ses fondements et sa forme. Il semble nécessaire dans ce contexte de clarifier le concept de formation à distance.

Le Comité de Liaison Interordres en Formation à Distance (CLIFAD) (2010:1) définit la FAD comme "un dispositif de formation comportant un ensemble de moyens organisés pour atteindre les objectifs d'un cours ou d'un programmes. ". Dans cette définition, la FAD va au-delà de la distance géographique qui sépare l'enseignant et l'apprenant. La définition met aussi l'accent sur les notions d'autonomie dans le processus d'apprentissage et de dispositifs (acteurs: enseignant/apprenants et outils techniques: informatique, internet, téléphone, médias) mis en place pour assurer la formation à distance.

Quant à Deschênes et à Maltais (2006: 16), la FAD se réfère à "une pratique éducative privilégiant une démarche d'apprentissage qui rapproche le savoir de l'apprenant. Dans un cours à distance, on cherche ainsi à réduire la distance spatiale et/ou temporelle séparant les deux". Pour ces derniers, la définition proposée tient compte de la situation d'éloignement comme le fait d'ailleurs CLIFAD qui parle de la séparation de l'enseignant et l'apprenant. Selon L'Université Laval (UL, 2016: 4), la FAD se conçoit comme "un système de formation qui permet à un étudiant d'apprendre seul ou en situation de collaboration, à l'aide de matériel didactique approprié, par différents moyens de communication et avec le soutien à distance de l'enseignant et de personnes-ressources". La définition que donne l'université Laval est intéressante parce qu'elle englobe les supports pédagogiques médiatisés mis en œuvre et de mode de formation et l'absence physique des acteurs.

En faisant référence aux définitions ci-hauts citées, nous estimons que la FAD peut être définie comme un mode de formation qui s'opère à distance par divers dispositifs dont les caractéristiques définitoires sont la distance physique, la distance temporelle et la médiation de la technologie (les médias audiovisuels et l'informatique, etc.). II nous semble nécessaire de faire le point sur les dispositifs mis en place comme l'ensemble d'acteurs (apprenants, tuteurs et responsables de formation) qui constituent un élément non négligeable à prendre en compte lors qu'on parle de la FAD. A ceux-ci s'ajoutent les outils techniques (ressources pédagogiques, outils d'échange et plateformes).

Quant à la formation en ligne dite aussi l'apprentissage en ligne ou l'e-learning, qui fait partie intégrante de la FAD, elle n'est pas une nouvelle conception dans les technologies de l'éducation, mais ce qui est nouvelle ici c'est son application à grande échelle à 
la suite de la crise sanitaire de Covid-19._Dans son avis sur la formation en ligne, la Commission des Communautés Européennes (2001:2) la définit comme "l'utilisation des nouvelles technologies multimédias de l'Internet pour améliorer la qualité de l'apprentissage en facilitant d'une part l'accès à des ressources et à des services, d'autre part les échanges et la collaboration à distance". De cette façon, la formation en ligne est l'usage des technologies de l'information et de la communication à des fins éducatives ce qui permet de dire qu'elle a un lien de parenté avec la FAD. En ce sens, elle se conçoit comme un modèle avancé de la $F A D$, c'est dire la formation synchrone qui s'effectue en temps réel.

\subsection{Définition et types d'interaction}

Pour Marc et Picard (2002: 189), I'interaction est "une action mutuelle en réciprocité de plusieurs éléments". Quant au Petit Robert (2005: 1385), il définit l'interaction comme une action réciproque. De son côté, le Cadre Commun Européen (2005:18) voit "dans l'interaction au moins deux acteurs participent à un échange oral et/ou écrit et alternent les moments de production et de réception qui peuvent même se chevaucher dans les échanges oraux." De ces définitions, nous précisons que les notions de réciprocité et de mutualité sont des exigences au processus d'interaction. Dans un contexte de formation que ce soit présentiel ou distanciel, elles sont à la base d'une communication réussie qui vise à assurer l'échange d'idées, de dialogues et d'activités entre les parties au processus d'apprentissage (enseignant et apprenants). Pour ce qui concerne les types d'interactions, d'après Moore (1989), il y en a trois:

1. L'interaction enseignant/apprenant. II comprend divers modes de communication, tels que l'échange de messages privés et la discussion de groupe, qui créent un dialogue et un échange d'informations entre les étudiants et les enseignants pour soutenir la motivation et l'apprentissage.

2. L'interaction apprenant/contenu. II s'agit de l'interaction entre l'apprenant et le matériel pédagogique. Cela implique que l'apprenant réfléchit activement à un contenu particulier afin de comprendre l'information. L'apprenant peut être engagé individuellement ou en groupe à travers des questions, des exercices et des activités qui stimulent la réflexion et l'interaction constructive avec le matériel.

3. L'interaction apprenant/apprenant. Ce type d'interaction comprend l'échange d'information et d'idées et le dialogue qui s'établit entre les étudiants dans un cou

\section{Méthodologie}

\subsection{Profil des participants}

L'échantillon étudié se compose de 20 apprenants étant essentiellement des étudiants au Département d'Anglais (College of Science and Arts, Turaif Campus, Northern Border University, KSA). Il s'agit donc des étudiants spécialisés en anglais enclassés en $3^{\text {ème }}$ niveau. Ils étaient tous des garçons d'âges différents, variés de 17 à 19 ans.

II importe de signaler que tous les apprenants ici sont arabophones, c'est-à-dire qu'ils ont l'arabe comme langue maternelle, nationale, de scolarisation, de médias etc., Pour leur vécu socioculturel, ils appartiennent à la même civilisation qui est la civilisation arabo-musulmane. Quant au vécu socio-économique, ils proviennent d'un milieu socio-économique favorisé.

Compte tenu du programme d'étude du Département d'anglais, au Collège des Sciences et des Lettres, au $3^{\text {ème }}$ semestre, les apprenants étudient le premier cours de français de base. II s'agit là d'un cours obligatoire de 30 heures par semestre. Les apprenants étaient donc, à la base, de niveau débutant.

\subsection{Démarche méthodologique}

Notre démarche méthodologique a essayé d'établir la relation entre la formation en ligne et le taux d'interaction en classe virtuelle, c'est-à-dire par l'approche qualitative, nous avons tenté d'apprécier l'impact de la formation en ligne sur les interactions actives et spontanées en classe virtuelle. A cet effet, une méthode descriptive analytique, basée principalement sur des observations directes effectuées en classe virtuelle, a été utilisée.

\subsection{Outil de la collecte des données}

Nous avons utilisé la technique d'observation directe telle qu'elle est définie par Arborio (2007) pour récolter les données auprès des sujets observés. L'observation dite structurée est basée sur un schéma d'observation qui nous a permis de catégoriser les observations réalisées en trois groups. L'ensemble d'observations a constitué le corpus que nous avons analysé pour apprécier le taux d'interactions active et spontanée,

\subsubsection{Grill1e d'évaluation}

Compte tenu de notre objectif particulier, nous avons construit une grille d'observation simplifiée pour les interactions directes effectuées dans les classes virtuelles ayant lieu tout au long du $2^{\text {ème }}$ semestre de l'année universitaire 2020/2021. Cette étude s'est basée sur une grille d'évaluation en tant qu'un instrument de mesure et d'observation pour apprécier les interactions directes. La grille, inspirée du schéma d'observation ci-haut cité, nous a permis de répertorier les interactions que nous avons observées afin 
d'en porter des jugements. Il s'agit alors d'une grille simple adaptée à l'objectif de l'étude. La grille comporte une échelle de notes de 1 à 10 permettant de discerner les niveaux de performance (élevé, moyen, faible). Elle est composée de trois critères, à savoir, le premier critère est le Professeur-apprenant, noté sur 5 points, le deuxième est l'Apprenant-contenu, noté sur 2.5 points et le dernier est l'Apprenant-apprenant, noté sur 2.5 points comme le montre Tableau 1 ci-après.

Tableau 1 détaille la grille d'évaluation des interactions

\begin{tabular}{|c|c|c|c|c|c|}
\hline \multicolumn{6}{|c|}{ Nombre total de séances: $\mathrm{N}$} \\
\hline \multirow[t]{2}{*}{ Apprenants } & \multicolumn{4}{|c|}{$\begin{array}{l}\text { Critères de performance et échelle de notes } \\
\text { Interactions observées/échelles de notes }\end{array}$} & \multirow[t]{2}{*}{ Commentaire } \\
\hline & $\begin{array}{l}\text { Professeur- } \\
\text { apprenant }\end{array}$ & $\begin{array}{l}\text { Apprenant- } \\
\text { contenu }\end{array}$ & $\begin{array}{l}\text { Apprenant- } \\
\text { apprenant }\end{array}$ & Total & \\
\hline$A_{n}$ & $(X * 0.45)=A$ & $(X * 0.23)=B$ & $(X * 0.23)=C$ & $\mathrm{D}$ & élevé moyen, faible \\
\hline
\end{tabular}

La variable $N$ désigne le nombre total de séances.

La variable $A_{n}$ désigne l'apprenant.

La variable $X$ désigne le nombre total d'interventions dans chaque critère.

Les variables $A, B$ et $C$ sont les notes issues de la multiplication de la variable $X$ par la note accordée à chaque critère soit 0.45 pour le $1^{\mathrm{er}}$ critère ou 0.23 pour les deux derniers critères.

La variable $D$ est le total calculé par la somme des notes accordées aux critères $(A+B+C=D)$

\subsection{Mesure et procédure}

L'observation s'est déroulée au $2^{\text {ème }}$ semestre 2020/2021. Nous avons au total 11 séances de tutorat auxquelles les étudiants ont interagi de manière différente. Nous avons procédé à l'observation directe portant sur l'interaction tripartite l'obejt de cette étude. Il est à noter que la mesure s'est effectuée tout au long de chaque séance, c'est-à-dire, dans chaque cours de français. A la fin de chaque séance, nous avons mis un petit plus $(+)$ dans la case de certains qui ont fait des efforts. Nous avons accordé 0.45 à la rubrique Professeur.-apprenant, et 0.23 aux rubriques Apprenant-contenu et Apprenant-apprenant respectivement selon que l'étudiant est ou non intervenu de façon active et spontanée. A la fin de 11 séances, nous avons comptabilisé le nombre total d'interventions à chaque rubrique soit $A, B$ et $C$. comme le montre Tableau 2 ci-dessous. II reste à signaler que nous avons pris en charge les classes de manière habituelle. De nombreuses activités observables ont été classées selon les trois catégories.

\section{Résultats et discussion}

Dans ce qui suit, nous présentons les résultats obtenus. Nous les présentons au moyen de tableau regroupant les trois types d'interactions dont il a été question plus haut. 
Tableau 2 récapitule les résultats obtenus

\begin{tabular}{|c|c|c|c|c|c|}
\hline \multicolumn{6}{|c|}{ Nombre total de séances: $\mathbf{1 1}$} \\
\hline \multirow[b]{2}{*}{ Apprenant } & \multicolumn{4}{|c|}{ Types d'interactions observés et échelle de notes } & \multirow[b]{2}{*}{ Commentaire } \\
\hline & $\begin{array}{l}\text { Prof.-apprenant } \\
5 \text { pts }\end{array}$ & $\begin{array}{l}\text { Apprenant-contenu } \\
2.5 \mathrm{pts}\end{array}$ & $\begin{array}{l}\text { Apprenant-apprenant } \\
2.5 \mathrm{pts}\end{array}$ & $\begin{array}{l}\text { Total } \\
10 \mathrm{pts}\end{array}$ & \\
\hline $\mathrm{A}_{1}$ & $(11 * 0.45)=5$ & $(11 * 0.23)=2.5$ & $(6.5 * 0.23)=1.5$ & 9 & élevé \\
\hline $\mathrm{A}_{2}$ & $(3 * 0.45)=1.35$ & $(5 * 0.23)=1.5$ & $(0 * 0.23)=0$ & 3 & faible \\
\hline$A_{3}$ & $(11 * 0.45)=5$ & $(11 * 0.23)=2.5$ & $(2 * 0.23)=0.5$ & 8 & moyen \\
\hline $\mathrm{A}_{4}$ & $\left(9^{*} 0.45\right)=4$ & $(11 * 0.23)=2.5$ & $(2 * 0.23)=0.5$ & 7 & moyen \\
\hline$A_{5}$ & $(9 * 0.45)=4$ & $(5 * 0.23)=1$ & $(0 * 0.23)=0$ & 5 & faible \\
\hline$A_{6}$ & $(11 * 0.45)=5$ & $(11 * 0.23)=2.5$ & $(2 * 0.23)=0.5$ & 8 & moyen \\
\hline $\mathrm{A}_{7}$ & $(9 * 0.45)=4$ & $(11 * 0.23)=2.5$ & $(2 * 0.23)=0.5$ & 6 & moyen \\
\hline$A_{8}$ & $(9 * 0.45)=4$ & $(9 * 0.23)=2$ & $(0 * 0.23)=0$ & 6 & moyen \\
\hline$A_{9}$ & $\left(11^{\star} 0.45\right)=5$ & $(11 * 0.23)=2.5$ & $\left(6.5^{*} 0.23\right)=1.5$ & 9 & élevé \\
\hline $\mathrm{A}_{10}$ & $(11 * 0.45)=5$ & $(9 * 0.23)=2$ & $(5 * 0.23)=1$ & 8 & moyen \\
\hline$A_{11}$ & $(11 * 0.45)=5$ & $(9 * 0.23)=2$ & $(2 * 0.23)=0.5$ & 8 & moyen \\
\hline$A_{12}$ & $(11 * 0.45)=5$ & $(11 * 0.23)=2.5$ & $(6.5 * 0.23)=1.5$ & 9 & élévé \\
\hline$A_{13}$ & $(8 * 0.45)=3.5$ & $(11 * 0.23)=2.5$ & $(5 * 0.23)=1$ & 7 & moyen \\
\hline $\mathrm{A}_{14}$ & $(11 * 0.45)=5$ & $(9 * 0.23)=2$ & $(0 * 0.23)=0$ & 7 & moyen \\
\hline$A_{15}$ & $(9 * 0.45)=4$ & $(9 * 0.23)=2$ & $(2 * 0.23)=0.5$ & 6 & moyen \\
\hline$A_{16}$ & $(9 * 0.45)=4$ & $(9 * 0.23)=2$ & $(0 * 0.23)=0$ & 6 & moyen \\
\hline $\mathrm{A}_{17}$ & $(11 * 0.45)=5$ & $(11 * 0.23)=2.5$ & $(6.5 * 0.23)=1.5$ & 9 & élevé \\
\hline$A_{18}$ & $(9 * 0.45)=4$ & $(11 * 0.23)=2.5$ & $(6 * 0.23)=1.5$ & 6 & moyen \\
\hline$A_{19}$ & $(11 * 0.45)=5$ & $(11 * 0.23)=2.5$ & $(2 * 0.23)=0.5$ & 8 & moyen \\
\hline $\mathrm{A}_{20}$ & $\left(11.5^{\star} 0.45\right)=5$ & $(9 * 0.23)=2$ & $(0 * 0.23)=0$ & 7 & moyen \\
\hline
\end{tabular}

- $\quad 0.45$ ou 0.23 note attribuée à chaque participation

- $\quad$ faible $=1$ à 5 , moyen $=6$ à 8 , élevé $=9$ à 10

A l'égard du $1^{\mathrm{er}}$ type d'interaction Professeur-apprenant, les résultats nous dévoilent que 11 des sujets observés ont obtenu 5 point sur 5 , soit $55 \%$, alors que 7 ont obtenu 4 points, soit $35 \%$, un seul sujet a obtenu 3.5 points, soit $5 \%$ et un autre a obtenu 2.5 points, soit $5 \%$. A propos de 2 ème type Apprenant-contenu, 11 du public observé ont obtenu 2.5 points sur 2.5 , soit $55 \%, 8$ ont obtenu 2 points, soit $40 \%$ et 1 apprenant a obtenu 1 point, soit $5 \%$. S'agissant du 3 ème type, Apprenant-apprenant, nous trouvons que 6 des sujets observés ont obtenu 1.5 points, soit $30 \%$ contre 2 qui ont obtenu 1 point, soit $10 \%$, 7 ont obtenu 0.5 soit $35 \%$ et 5 n'ont rien obtenu, 0 point soit $25 \%$.

En ce qui concerne l'ensemble des notes obtenues par les sujets observés, les résultats montrent que 4 sujets ont obtenu 9 points, soit $20 \%$, 5 ont obtenu 8 points, soit $25 \%$, 4 ont obtenu 7 points, soit $20 \%$, 5 a obtenu 6 points, soit $25 \%$, 1 sujet a obtenu 5 points, soit $5 \%$ et un autre a obtenu 3 points, soit $5 \%$ comme le montre Tableau 3 ci-dessous.

Tableau 3 résume les résultats en pourcentage

\begin{tabular}{|l|l|l|l|}
\hline $\mathbf{n}^{\mathbf{0}}$ & Nombre d'étudiants & Points obtenus & Pourcentage \\
\hline 1 & 4 & 9 & $20 \%$ \\
\hline 2 & 5 & 8 & $25 \%$ \\
\hline 3 & 4 & 7 & $20 \%$ \\
\hline 4 & 5 & 6 & $25 \%$ \\
\hline 5 & 1 & 5 & $5 \%$ \\
\hline 6 & 1 & 5 & $5 \%$ \\
\hline Total & 20 & & $100 \%$ \\
\hline
\end{tabular}

De ce qui précède, nous précisons que sur les 20 apprenants observés, 11 apprenants soit 55\% ont obtenu des notes inférieures au seuil de réussite qui est de 7.5 points (3, 5, 6 et 7 respectivement) contre 9 apprenants, soit $45 \%$, qui ont obtenu des notes supérieures à celle de passage (8 et 9 respectivement). Ceci nous permet de constater que le taux d'interactions de nos sujets est insatisfaisant comme le montre Tableau 4 ci-dessous. 
Tableau 4 détaille le taux de réussite

\begin{tabular}{|l|l|l|l|}
\hline $\mathbf{n}^{\mathbf{0}}$ & Nombre d'étudiants & $\mathbf{\%}$ & Commentaire sur le pourcentage \\
\hline 1 & 11 & $55 \%$ & inférieur au seuil de réussite fixé à 7.5 \\
\hline 2 & 9 & $45 \%$ & supérieur au seuil de réussite fixé à 7.5 \\
\hline
\end{tabular}

Pour se rendre compte du deuxième objectif de cette étude, nous serons amenés à analyser de manière descriptive les observations sous forme de pratiques langagières en mettant l'accent principalement sur les différents types d'interactions observés (Professeur-apprenant, Apprenant-contenu et Apprenant-apprenant.) dans toutes les séances. Notre analyse se fonde donc sur l'étude de corpus que nous avons recueilli.

\subsection{Interaction Professeur-apprenant}

L'analyse des interactions professeur-apprenant se porte sur les pratiques langagières comme nous l'avons cité plus haut. Les résultats montrent un nombre assez remarquable de ce type d'interaction ce qui illustre bien la réussite de ce type d'échange lors des interactions basées sur l'apprentissage interactif. Le taux élevé d'interaction est expliqué en partie par le fait que le professeur prenait le relais de l'interaction en s'appuyant ici sur l'apprentissage collaboratif pour achever les actes langagiers. En effet, les interactions observées se sont réalisées par la prise de parole et/ou le recours au chat sous diverses formes d'actes langagiers qui expriment, par exemple, la communication quotidienne comme le montre l'extrait ci-dessous (le professeur est codifié $\mathrm{P}$ et les apprenants sont codifiés E1, E2, E3 etc.)

P: Bonjour, vous allez bien?

E2: Bonjour Docteur, bien

\section{E8: Bonjour Monsieur}

Le thème autour duquel les échanges ont été centrés dans les interactions observées est la salutation (formelle et informelle), il s'agit là d'un acte langagier fréquent ce qui donne lieu à une interaction intéressante. Il est évident qu'au cours de ces interactions basées sur l'acte de salutation, les intervenants ont utilisé différentes pratiques langagières ce qui est un témoignage de la réalisation de l'objectif à atteindre. Le fait qui permet de signaler que les apprenants pouvant facilement interagir avec le professeur lors l'acte de communication leur est familier et habituel.

En effet, l'échange sous forme de questions guidées, qui est une autre forme d'interaction observée ici, vise à renforcer l'engagement des apprenants et à les rendre interactifs.

Une autre pratique langagière pouvant montrer l'échange sous forme de question guidée.

P: Comment vous appelez-vous?

E5: Je m'appelle Soud

P: bien Soud, vous avez quel âge?

E5: 19

P: Merci bien, c'est très gentil.

E5: de rien

Nous signalons que cet échange déroulé entièrement en français constitue un autre témoignage de la participation active et positive de l'apprenant dans cette situation d'apprentissage dite fréquente.

Voici un autre exemple qui illustre assez bien l'échange collaboratif lors d'une séance de collaboration. L'extrait qui suit est tiré d'un échange qui met aux prises le P. et l'E5.

P: Anas, comptez de 1 à 15 , s'il vous plait.

E5: un, deux, trois, cinq

P: Pardon, trois, quatre, cinq 
E5: Ok, trois, quatre, cinq

Nous avons ici un acte de parole accompli centré sur les chiffres qui jouent le rôle d'un simple déclencheur d'interaction. Ceci donne lieu à l'engagement dans le processus d'interaction par la prise de parole où l'enseignant a interagi et travaillé de façon collaborative.

De manière générale, nous constatons que lors de ces observations, nous avons recensé un nombre remarquable de ces types d'échange où les apprenants se sont engagés aux réponses des questions guidées.

\subsection{Interaction apprenant-contenu}

l'interaction Apprenant- contenu est la forme la plus importante d'interactions parce qu'elle consiste à renforcer l'engagement et la motivation des étudiants vis-à-vis du matériel pédagogique et permettre d'améliorer leur performance. Ce type d'interaction, comme nous le savons, fait référence au contenu d'apprentissage qui comprend différentes activités mises en pratique. Les observations montrent que les apprenants étaient dans une certaine mesure actifs à ce niveau-là et ont interagi de temps en temps avec le contenu d'apprentissage à travers les diverses activités d'apprentissage sur la plateforme pédagogique Blackboard. Les interactions observées consistaient à assurer la lecture dirigée, l'écoute et la compréhension orale/écrite relatives aux activités aux documents circulés dans le contenu d'apprentissage. Nous en citons quelques-unes, à titre d'exemple, bon nombre d'étudiants ont interagi avec le texte de présentation ci-dessous:

Je m'appelle Nora, je vis en Tunisie, je suis tunisienne. J'ai cinquante-cinq ans. Je suis médecin. Ma passion, c'est la peinture et j'ai un rêve: visiter les musées de Paris, le Louvre et tous les autres.

\section{Choisissez Vrai ou Faux.}

1. Nora est algérienne (). 2. Elle a 35 ans (). 3. Elle est passionnée de la peinture (). 4. Elle rêve de visiter les musées de Toronto ( ). 5. Elle est étudiante en médicine ().

Leur interaction se manifeste dans la lecture individuelle du texte et la réponse aux exercices de type Vrai/Faux. Certains étudiants ont également interagi avec d'autres types d'exercices tels que les QCMs, associer, remplir les blancs etc. Les quiz en ligne sont aussi présents dans les activités basées sur l'interaction apprenant-contenu. Les observations en montrent quelques-uns comprenant des warm-up quiz faits au début des cours et des quiz périodiques réalisés à la fin des séquences d'apprentissage.

\subsection{Interaction apprenant-apprenant}

L'interaction apprenant-apprenant est censé se réaliser de manière naturelle quand les étudiants écoutent les commentaires des autres, se posent des questions et établissent des relations grâce à des contacts fréquents. II apparaît que de nombreux apprenants manquent les compétences de base pour interagir avec leurs pairs. En fait, nous avons à peine observe des interactions entre pairs. Certains ont interagi avec succès en posant des questions à leurs pairs comme le montre l'extrait suivant :

\section{E1 : ça va Anas?}

E5: uhh, ça va, oui, oui, ça va et toi ?

E1 : ça va bien merci.

D'autres ont essayé d'entamer un dialogue guidé axé sur le thème de présentation étant le thème le plus abordé, mais sans vain parce que leurs pairs étaient réticents à interagir et refusaient de prendre le relais de la communication.

En gros, nous constatons que l'interaction entre pairs est le maillot faible des interactions observées chez nos apprenants.

\section{Conclusion}

En guise de conclusion, nous précisons que sur la base de l'analyse des données récoltées par le biais des observations systématiques sur les interactions et des résultats obtenus, nous estimons que cette étude a permis de constater que certains étudiants ont pu facilement interagir avec le professeur et le contenu ce qui favorise davantage les interactions en classe virtuelle. Par contre, beaucoup n'ont pas pu interagir entre eux ce qui aurait des effets négatifs sur le processus d'apprentissage en classe virtuelle.

L'interaction, la communication efficace, l'échange d'idées et la relation y établie entre les pairs peuvent favoriser l'interaction. De plus, l'interaction efficace contribue au développement des étudiants sur le plan linguistique car elle favorise l'acquisition du 
savoir-être, en ce sens, elle leur permet de prendre confiance en eux, les rend à être autonomes. Elle favoriser aussi la communication en permettant de développer chez l'apprenant des savoirs-faire.

Cette étude, même si elle n'est pas exhaustive, permet de proposer quelques pratiques de classe pour favoriser les interactions en classe virtuelle:

- Les stratégies pédagogiques interactives nous semble important, voire fondamentale pour renforcer les interactions dans l'univers de la formation en ligne. En ce sens, l'on peut enseigner de façon interactive par la création des moments d'échange, d'interaction, d'engagement, et de collaboration.

- Le recours aux cours interactifs peut activement engager et impliquer les étudiants dans un cours magistral en introduisant des activités qui permettent aux étudiants d'appliquer ce qu'ils ont appris.

- $\quad$ Le concept de Temps de Questions (Question Time) est d'une grande utilité. Le professeur peut consacrer les dix dernières minutes à chaque cours à Question Time. Lors de cette période, les étudiants peuvent poser de différentes questions interactives dans la boîte de chat (chat box), alors le professeur se met à les répondre le fait qui favorise les interactions.

- L'idée d'exercices interactifs générés en ligne permet de varier les activités d'apprentissage le qui favorise davantage les interactions en classe virtuelle.

- Un générateur d'exercices en ligne peut jouer un grand rôle dans la création des activités interactives telles que QCM, Quiz, des textes à trous, des dictées, etc. .

Compte tenu des limites de cette étude, nous n'avons pas pu analyser l'ensemble de ce sujet très vaste. Cependant, il nous semblerait intéressant, dans l'avenir, d'identifier les facteurs qui entrent en jeu et affectent les interactions dans l'univers de la formation en ligne. 


\title{
Les effets de la formation en ligne sur les interactions en classe virtuelle
}

\author{
"Etude de cas, Faculté des Sciences et des Lettres, Campus de Turaif, NBU, KSA" \\ Omer Ahmed Omer, College of Sciences and Arts (Turaif), NBU, KSA, \\ Omar.Ahmed@nbu.edu.sa \\ Nadra Abd Allah Ali, Faculté des Lettres, Université de Khartoum, Soudan \\ Nadraelfadil2003@yahoo.fr
}

Résumé

La présente étude qualitative se propose d'apprécier et analyser de manière descriptive les effets de la formation en ligne sur les interactions en classe virtuelle de FLE dans le but de renforcer l'engagement des apprenants dans ce type de classe. La problématique qui se fait sentir chez nous, en tant que professeurs, est le manque quasi total d'interactions spontanée et active en classe virtuelle ce qui peut défavoriser de manière inquiétante le dynamisme du processus d'apprentissage. L'étude s'est basée sur des données issues

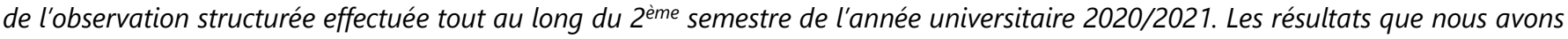
obtenus montrent que le taux général d'interaction est insatisfaisant notamment le taux d'interaction entre pairs qui est le pire. Certaines pratiques de classe ont été recommandées pour favoriser les interactions au sein de classe virtuelle. Une étude ultérieure pourrait examiner les facteurs qui entrent en jeu et affectent les interactions dans l'univers de la formation en ligne.

Mots-clés: formation en ligne, interaction, classe virtuelle, observation structurée. 


\section{References}

[1] Arborio, A. (2007). L'observation directe en sociologie : quelques réflexions méthodologiques à propos de travaux de recherches sur le terrain hospitalier. Recherche en soins infirmiers, n 90, 26-34. https://doi.org/10.3917/rsi.090.0026.

[2] CLIFAD (2010). Définition de la formation à distance, réf. d'avril 2020, http://clifad.qc.ca/ definition-de-la-formation-a-distance.html.

[3] Commission des Communautés Européenne, e-Learning - Penser l'éducation de demain, Communication de la Commission; Bruxelles, 2000-05-24 COM(2000).

[4] Commission des Communautés Européennes: Plan d'action e-learning Penser l'éducation de demain, Communication de la Commission au Conseil et au Parlement Europeen, Bruxelles, le 28.3.2001 COM(2001).

[5] Conseil de l'Europe, Cadre européen commun de référence pour les langues, Paris, Didier, 2005.

[6] Conseil de l'Europe, Cadre européen commun de référence pour les langues : apprendre, enseigner, évaluer, Unité des Politiques linguistiques, Strasbourg www.coe.int/lang-CECR

[7] Deschênes, A. J. et Maltais. M. (2006): Formation à distance et accessibilité. Télé-université, edutice-00078809.https://edutice.archivesouvertes.fr/edutice-00078809/document

[8] Glikman, V. (2002). Des cours par correspondance au « e-learning »: Panorama des formations ouvertes et à distance. Paris cedex 14, France: Presses Universitaires de France. https://doi.org/10.3917/puf.glik.2002.01

[9] Le Petit Robert de la langue française (2005), Editions Le Robert 2005

[10] Marc, E. \& Picard, D. (2002). Interaction. Dans : Jacqueline Barus-Michel éd., Vocabulaire de psychosociologie (pp. 189-196). Toulouse, France: https://www.cairn.info/vocabulaire-de-psychosociologie--9782749206851-page-189.htm

[11] Moore, M.G. (1989). Three types of interaction. American Journal of Distance Education 3(2)

[12] Université L (2016). Politique de la formation à distance, Québec, L'Université, 8 p., réf. d'avril 2020,

https://www.ulaval.ca/fileadmin/Secretaire general/Politiques/Politique de la formation a distance CU-2016-57.pdf. 